\title{
Corrigendum
}

\section{Corrigendum to "Comparison of Different Histone Deacetylase Inhibitors in Attenuating Inflammatory Pain in Rats"}

\author{
Jing Zhou, ${ }^{1}$ Yu Mao, ${ }^{2,3}$ Xuesheng Liu, ${ }^{2}$ Erwei Gu $\mathbb{D}^{2},{ }^{2}$ Zhi Zhang, ${ }^{3}$ and Wenjuan Tao $\mathbb{D}^{4}$ \\ ${ }^{1}$ Department of Head-neck and Breast Surgery, Western district of the First Affiliated Hospital, \\ University of Science and Technology of China, Hefei City, Anhui 233004, China \\ ${ }^{2}$ Department of Anesthesiology, First Affiliated Hospital of Anhui Medical University, Jixi Road 218, Hefei City, Anhui, China \\ ${ }^{3}$ School of Life Sciences, University of Science and Technology of China, Huangshan Road 443, Hefei City, Anhui, China \\ ${ }^{4}$ School of Basic Medical Sciences, Anhui Medical University, Meishan Road 81, Hefei City, Anhui, China \\ Correspondence should be addressed to Yu Mao; maoyu163flying@163.com \\ Received 18 May 2021; Accepted 18 May 2021; Published 29 May 2021 \\ Copyright (c) 2021 Jing Zhou et al. This is an open access article distributed under the Creative Commons Attribution License, \\ which permits unrestricted use, distribution, and reproduction in any medium, provided the original work is properly cited.
}

In the article titled "Comparison of Different Histone Deacetylase Inhibitors in Attenuating Inflammatory Pain in Rats" [1], the order of the authors' list was incorrect in the original publication and the correct order of the authors' list is shown above.

\section{Authors' Contributions}

Jing Zhou and Yu Mao contributed equally to this study.

\section{References}

[1] Y. Mao, J. Zhou, X. Liu, E. Gu, Z. Zhang, and W. Tao, "Comparison of different histone deacetylase inhibitors in attenuating inflammatory pain in rats," Pain Research and Management, vol. 2019, Article ID 1648919, 10 pages, 2019. 